\title{
Recommendations for the treatment of hepatitis $C$ issued by the Polish Group of HCV Experts - 2016
}

\author{
Polish Group of HCV Experts: Waldemar Halota, Robert Flisiak, Anna Boroń-Kaczmarska, Jacek Juszczyk, \\ Piotr Małkowski, Małgorzata Pawłowska, Krzysztof Simon, Krzysztof Tomasiewicz
}

Address for correspondence

Prof. Robert Flisiak, Department of Infectious Diseases and Hepatology, Medical University of Białystok, 14 Żurawia St., 15-540 Bialystok, Poland, e-mail: robert.flisiak@umb.edu.pl

\section{Introduction}

Diseases of hepatitis C virus (HCV) aetiology are rarely diagnosed on the basis of the clinical picture, as their course is usually asymptomatic or only mildly symptomatic for many years. Consequently, diagnosis is frequently preceded by an incidental detection of laboratory markers indicative of $\mathrm{HCV}$ infection. Studies conducted in Poland in recent years have shown that anti-HCV antibodies are found in $0.9-1.9 \%$ of the Polish population, depending on the study population and the methodology applied. The studies have consistently confirmed the presence of HCV-RNA in the blood, indicating active infection, at $0.6 \%$. The figure corresponds to approx. 200,000 adult Poles who require immediate diagnosis and treatment. The number of patients diagnosed during the period of HCV therapy availability is estimated to be approx. 35,000, which is equivalent to the detection rate of $17.5 \%$ [1-3]. Hepatitis C virus genotype (GT) 1 is the most prevalent one among the Polish population: it is found in $80 \%$ of all infected individuals, the most common (98\%) sub-genotype being GT1b. GT3 (14\%) and GT4 (5\%) are less common, and infections with genotypes 2, 5 and 6 may be diagnosed sporadically [4].

Around $20-40 \%$ of acute infections are thought to resolve spontaneously. Chronic HCV infection only manifests itself after many years, and one in five patients develop advanced pathological changes in the liver including cirrhosis or hepatocellular carcinoma (HCC). Hepatitis C virus infection also induces a number of extrahepatic syndromes, most typically cryoglobulinemia which gives rise to clinical manifestations in 5-25\% of cases, and B-cell non-Hodgkin lymphoma (B-NHL) [5].
All patients with chronic HCV infection should receive treatment. The sooner the therapy is initiated, the better the outcome and the lower the cost. However, if access to therapy is restricted, priority should be given to those patients whose $\mathrm{HCV}$ infection, in the assessment of an infectious diseases specialist, can lead to a deterioration of the quality of life or death within a short time. The aims of treatment are to eliminate $\mathrm{HCV}$ infection and, consequently, to stop or reverse histological lesions, and to reduce the risk of development of hepatocellular carcinoma and prevent the spread of the infection to other people [6, 7].

\section{Acute HCV infection}

The only objective criterion in the diagnosis of acute hepatitis C (AHC) is the identification of AHC-associated laboratory markers (elevation of alanine aminotransferase activity, presence of anti-HCV and/or HCVRNA) in patients whose prior $\mathrm{HCV}$ tests were negative or in patients who had a documented exposure to $\mathrm{HCV}$ infection. In other cases, the diagnosis of acute hepatitis $\mathrm{C}$ may be inconclusive. Importantly, while HCV-RNA is detectable as early as 1-3 weeks after exposure, antiHCV antibodies cannot be detected until 4-10 weeks after infection. Following the onset of the first clinical manifestations, if they appear, anti-HCV antibodies are present in just $50-70 \%$ of infected patients, and the percentage exceeds $90 \%$ after as long as three months. In fact, some patients do not develop anti-HCV antibodies at all. In such cases, the basis for diagnosing HCV infection is the presence of HCV-RNA in the blood.

Therapy may be initiated if HCV-RNA is still detectable 12 weeks after the diagnosis of $\mathrm{HCV}$ infection. In such cases, patients receive pegylated interferon alpha (PegIFN $\alpha$ ) 2a or $2 b$ in monotherapy for 12 weeks. 
In patients with $\mathrm{HIV}-\mathrm{HCV}$ co-infection, the duration of therapy should be extended to 24 weeks, and ribavirin (RBV) should be added $[6,7]$.

An alternative therapeutic option is interferon-free treatment which can be initiated 24 weeks after the diagnosis of HCV infection. In this case, therapy should be provided in conformity with rules laid down for chronic HCV infection [7].

\section{Chronic HCV infections}

The basic criterion for diagnosing chronic diseases of HCV aetiology is the presence of HCV-RNA (in blood serum, liver tissue or peripheral blood mononuclears) persisting for at least six months in a patient with markers of liver disease or an extrahepatic manifestation of the infection. Hepatitis $\mathrm{C}$ virus infection in the liver can result in changes described as chronic hepatitis $\mathrm{C}$ and cirrhosis or hepatocellular carcinoma. In HCV-infected patients diagnosed with cirrhosis, the requirement to wait six months before the initiation of therapy does not need to be met. The process of assessing eligibility for treatment should involve the determination of viral genotype, and if genotype 1 is detected, also the determination of subgenotype (GT1a or GT1b) and assessment of the stage of liver fibrosis. Treatment should be monitored by assaying the concentration of HCV-RNA using techniques with the limit of detection $\leq 15 \mathrm{IU} / \mathrm{ml}$.

\section{General recommendations}

The therapeutic regimen must be selected on the basis of the current availability, efficacy and safety profile. Patients should be provided with clear information about the duration of therapy, potential adverse reactions associated with each drug, importance of adhering to the prescribed treatment regimen and rules governing therapy continuation and interruption.

\section{Recommended drugs}

Table 1 lists drugs registered in any country worldwide, and particularly drugs approved by the EMA (European Medicines Agency) or FDA (Food and Drug Administration), as they are likely to be available on the Polish market in the near future. Using other direct acting antivirals (DAA) drugs is also acceptable, provided that they are approved according to their SPC (summary of product characteristics).

\section{Assessment of liver fibrosis}

The degree of liver fibrosis should be assessed on a 5-point scale from 0 to 4 using liver elastography performed with a technique allowing to evaluate the stiffness of the liver tissue in $\mathrm{kPa}$ (share wave elastography, transient elastography), or liver biopsy. If coexisting liver diseases of a different aetiology are suspected, and

Table 1. Dosage regimens of drugs included in the recommendations (drugs in groups are listed alphabetically)

\begin{tabular}{|c|c|c|c|}
\hline Drug category & Class & Drugs & Daily dosage \\
\hline \multirow[t]{12}{*}{ Direct acting antivirals (DAA) } & NS3 inhibitors (protease) & Asunaprevir (ASV) & $200 \mathrm{mg} /$ day in 2 doses \\
\hline & & Boceprevir (BOC) & $2,400 \mathrm{mg} /$ day in 3 doses \\
\hline & & Grazoprevir (GZR) & $100 \mathrm{mg} /$ day in 1 dose* \\
\hline & & Paritaprevir (PTV) & $150 \mathrm{mg} /$ day in 1 dose $^{* *}$ \\
\hline & & Simeprevir (SMV) & $150 \mathrm{mg} /$ day in 1 dose \\
\hline & & Telaprevir (TVR) & $2,250 \mathrm{mg} /$ day in 2 doses \\
\hline & NS5B inhibitors (polymerase) & Dasabuvir (DSV) & $500 \mathrm{mg} /$ day in 2 doses \\
\hline & & Sofosbuvir (SOF) & $400 \mathrm{mg} /$ day in 1 dose $\mathrm{s}^{* *}$ \\
\hline & NS5A inhibitors & Daclatasvir (DCV) & $60 \mathrm{mg} /$ day in 1 dose \\
\hline & & Elbasvir (EBR) & $50 \mathrm{mg} /$ day in 1 dose* \\
\hline & & Ledipasvir (LDV) & $90 \mathrm{mg} /$ day in 1 dose ${ }^{* *}$ \\
\hline & & Ombitasvir (OBV) & $25 \mathrm{mg} /$ day in 1 dose ** \\
\hline \multirow[t]{2}{*}{ Interferons } & Pegylated interferons $\alpha$ (PegIFN $\alpha)$ & PegIFNa2a & 180 g/week $\#$ \\
\hline & & PeglFN $\alpha 2 b$ & $1.5 \mu \mathrm{g} / \mathrm{kg} /$ week $^{\#}$ \\
\hline Others & & Ribavirin (RBV) & 1,000 or $1,200 \mathrm{mg}$ at $\mathrm{BW}<75 \mathrm{~kg}$ or $>75 \mathrm{~kg}^{\prime \prime \#}$ \\
\hline
\end{tabular}

* GZR and EBR are available in one tablet

**PTV and OBV are available in one tablet with ritonavir ( $r$ )

***SOF is available alone or in one tablet with LDV

\#PegIFNo 2a in children - 65-180 $\mu \mathrm{g} /$ week depending on the body surface area according to summary of product characteristics

\#PegIFNa2b in children $-65 \mu \mathrm{g} / \mathrm{m}^{2} /$ week

${ }^{\# \#} \mathrm{RBV}$ in children $-15 \mathrm{mg} / \mathrm{kg}$ 
the result of a non-invasive examination is not consistent with the patient's clinical condition or discrepancies are shown between the results of different non-invasive tests, liver biopsy is recommended (unless there are contraindications to the procedure). Liver biopsy findings are considered conclusive in such cases $[7,8]$. If both liver biopsy and elastography are contraindicated, the patient can be assessed on the basis of results obtained in one of serum tests available, the simplest of which is APRI [9].

\section{Assessment of treatment efficacy}

Treatment can be considered effective if no HCVRNA is detected in blood at week 24 after the completion of therapy, signifying the achievement of sustained virological response (SVR24). The efficacy of therapy should be assessed by methods ensuring the detection level of $\leq 15 \mathrm{IU} / \mathrm{ml}[6,7]$.

\section{Direct acting antivirals resistance}

Due to the risk of selection of resistant strains (resistance associated variants - RAV) DAA monotherapy is unacceptable. Interferon-free therapy should combine between two and four NS3, NS5A and NS5B inhibitors, possibly in conjunction with RBV. Resistance associated variants have the greatest practical significance for NS5A due to the durability of resistance and its widespread nature. Patients with no prior interferon-free treatment do not require testing for RAV.

Following unsuccessful dual DAA therapy, GT1binfected patients can be retreated with more than two DAA with different mechanisms of action, without testing for RAV.

The test is necessary prior to planned retherapy in patients infected with:

- GT1b after the failure of a therapeutic regimen consisting of more than two different DAA,

- GT other than $1 \mathrm{~b}$ after the failure of a therapeutic regimen containing NS5A inhibitor.

Testing for RAV should be performed by population sequencing with a sensitivity of detecting resistant variants $>15 \%$. In the light of the current knowledge, deep sequencing (with a sensitivity level $>1 \%$ ) is not beneficial for the optimization of therapy [10].

\section{Monitoring for hepatocellular carcinoma}

$\mathrm{HCV}$-infected individuals, especially with coexisting cirrhosis, should be systemically monitored for the development of HCC by liver ultrasound and, if necessary, also by evaluating $\alpha$-fetoprotein (AFP) levels. Assays should be performed at 24-week intervals even after the completion of successful therapy. Evaluation of AFP concentration should not be used alone for the early diagnosis of HCC. However, it may be useful in determining the prognosis of previously diagnosed cancer and in therapy monitoring. If a cancer lesion is suspected, four-phase computed tomography (CT) scan with contrast or magnetic resonance imaging (MRI) with contrast is recommended. Contrast-enhanced ultrasound, however, is not recommended for the routine diagnosis of $\operatorname{HCC}[6,7$, 11]. Both ultrasound and CT scan and/or MRI scan should be performed by radiologists experienced in liver imaging $[7,11]$.

\section{Hepatitis B virus and HIV co-infections}

The therapy of HBV-HCV or HIV-HCV co-infection is the same as the treatment regimen indicated in $\mathrm{HCV}$ monoinfection. As with other comorbidities, patients on antiretroviral therapy should be considered for possible drug interactions.

\section{Kidney failure}

Patients with eGFR $\geq 30 \mathrm{ml} / \mathrm{min} / 1.73 \mathrm{~m}^{2}$ should receive treatment in line with general principles of HCV therapy. When selecting optimum therapy for patients with severe renal dysfunction $(e G F R<30 \mathrm{ml} /$ $\mathrm{min} / 1.73 \mathrm{~m}^{2}$ ), especially on haemodialysis treatment, the preferred option is interferon-free treatment. Ribavirin should be avoided in this group of patients, and renal function should be checked regularly, especially in patients receiving sofosbuvir.

\section{Liver transplantation}

The precondition for protecting transplanted liver from the relapse of $\mathrm{HCV}$ infection is the suppression of viraemia to undetectable levels at least a month prior to the transplantation procedure. Consequently, therapy should be initiated as early as possible after the approval for liver transplantation. However, if the expected period until the transplantation procedure is so short that it does not guarantee effective suppression of the virus, anti-HCV treatment should not be instituted, and the patient should be closely monitored for the recurrence of viraemia in the transplanted liver. If this happens, interferon-free therapy should be initiated within a month of HCV-RNA detection. Patients undergoing liver transplantation during anti-HCV therapy should continue treatment for 12 weeks post procedure. Before starting DAA therapy, potential drug 
interactions must be considered to determine whether a dose adjustment of immunosuppressive drugs is necessary $[12,13]$.

\section{Direct acting antivirals drug interactions}

Before HCV treatment is initiated, potential interactions with other drugs used by the patient must be assessed to determine their potential for affecting the efficacy, dosage or safety of treatment. If interactions develop, previously used drugs should be replaced with safe alternatives or a different $\mathrm{HCV}$ treatment regimen should be considered. The above also applies to comorbidities, e.g. renal dysfunction, in which sofosbuvir treatment may be contraindicated. Most uncertainties about drug interactions can be resolved by checking the website at www.hep-druginteractions.org [13].

\section{Patients with decompensated cirrhosis}

Therapy in patients with a history of hepatic encephalopathy incidents, ascites, Child-Pugh scores B and $\mathrm{C}$ and in patients after liver transplantation should be conducted under constant monitoring in medical centres with experience in the treatment of patients with decompensated cirrhosis, with a possibility of immediate hospitalization and assessment of eligibility for liver transplantation. Some therapies should not be provided to patients with Child-Pugh score C. Detailed information is available under Specific recommendations.

\section{Specific recommendations}

The basic criterion determining the therapeutic approach is the HCV genotype (Table 2).

\section{HCV genotype 1 infections}

Optimal treatment of GT1 infections in previously untreated patients and following failure of treatment with PegIFN $\alpha+$ RBV or triple therapies with BOC or TVR is based on OBV/PTV/r + DSV or SOF/LDV - in some cases combined with RBV. Previously untreated GT1b-infected individuals can be considered for treatment using ASV + DCV. Interferon-based therapy in GT1-infected patients is not recommended because it is associated with a less favourable efficacy and safety profile than interferon-free therapy.

\section{Ombitasvir/paritaprevir/ritonavir + dasabuvir}

Patients infected with HCV subgenotype 1b, irrespective of previous treatment (also following unsuccessful triple interferon-based therapy) and the stage of fibrosis (also in cirrhosis) should receive OBV/PTV/r + DSV for 12 weeks without the need of adding RBV. In patients infected with HCV subgenotype la, the regimen is supplemented with RBV, and in cases of cirrhosis of this aetiology the duration of treatment is additionally extended to 24 weeks. The therapeutic regi-

Table 2. Recommended therapies and their durations depending on the viral genotype in adult patients. Detailed information on the therapies and therapeutic management in children are included in the text. The options recommended as first-line therapies are underlined

\begin{tabular}{|c|c|c|c|c|c|c|c|}
\hline & GT1a & GT1b & GT2 & GT3 & GT4 & GT5 & GT6 \\
\hline $\mathrm{OBV} / \mathrm{PTV} / \mathrm{r}+\mathrm{DSV}+\mathrm{RBV}$ & $\underline{12-24 \text { weeks }}$ & & & & & & \\
\hline OBV/PTV/r + DSV & & $\underline{12 \text { weeks }}$ & & & & & \\
\hline OBV/PTV/r + RBV & & & & & $\underline{12-24 \text { weeks }}$ & & \\
\hline ASV + DCV & & 24 weeks & & & & & \\
\hline$S O F / L D V \pm R B V$ & $\underline{12-24 \text { weeks }}$ & $12-24$ weeks & & 24 weeks & $\underline{12-24 \text { weeks }}$ & $\underline{12-24 \text { weeks }}$ & $\underline{12-24 \text { weeks }}$ \\
\hline SOF + PegIFN $\alpha+$ RBV & & & & $\underline{12 \text { weeks }}$ & 12 weeks & 12 weeks & 12 weeks \\
\hline $\mathrm{SOF}+\mathrm{RBV}$ & & & $\underline{12-24 \text { weeks }}$ & 24 weeks & 24 weeks & 24 weeks & 24 weeks \\
\hline$S O F+D C V+R B V$ & & & 24 weeks & 24 weeks & & & \\
\hline GZR/EBR & $12-16$ weeks & 12 weeks & & 12 weeks* & $12-16$ weeks & & \\
\hline PegIFN $\alpha+$ RBV & 48 weeks* * & 48 weeks** & 24 weeks $^{* * *}$ & 24 weeks*** & 48 weeks** & & \\
\hline
\end{tabular}

*In combination with SOF

** Only recommended in children

***Recommended in children, in adults subject to consideration

" + " Combined therapy with drugs available as separate preparations

"I" Combined therapy with drugs available as one combined preparation

" \pm "Addition of RBV depending on indications; details are provided in the text

OBV - ombitasvir, PTV - paritaprevir, /r/ - ritonavir, DSV - dasabuvir, RBV - ribavirin, ASV - asunaprevir, DCV - daclatasvir, SOF - sofosbuvir, PegIFN $\alpha$ - pegylated interferon $\alpha$,

GZR - grazoprevir, EBR - elbasvir 
men in patients infected with HCV of an unknown or inconclusive GT1 subgenotype or with mixed GT1a/1b infection should be the same as in patients infected with $\mathrm{HCV}$ genotype 1a. After liver transplantation, a 24-week OBV/PTV/r + DSV + RBV therapy should be initiated, regardless of HCV subtype. OBV/PTVr + DSV should not be used in patients with Child-Pugh score C [14-16].

\section{Sofosbuvir/ledipasvir}

Previously untreated patients without cirrhosis should continue SOF/LDV treatment for 12 weeks. Reducing the duration of therapy to 8 weeks does not seem to be fully justified by clinical practice. Patients who have failed previous therapy (including triple interferon-based therapy), with cirrhosis (also decompensated), and after liver transplantation should receive SOF/LDV + RBV for 12 weeks, and if RBV cannot be used, SOF/ LDV treatment should be extended to 24 weeks [17].

\section{Asunaprevir + daclatasvir}

ASV + DCV can be considered in previously untreated patients infected with genotype $1 \mathrm{~b}$. The duration of therapy is 24 weeks [18-20].

\section{Grazoprevir + elbasvir}

GZR/EBR therapy in GT1-infected patients should last 12 weeks. In patients who have failed triple interferon-based therapy, treatment should be supplemented with RBV.

The duration of GZR/EBR + RBV treatment should be extended to 16 weeks in GT1a-infected patients, if RAV to NS5A are detected [21].

\section{Pegylated interferon $\alpha+$ ribavirin}

The therapy is recommended in children after 3 years of age for a total of 48 weeks $[22,23]$.

\section{Failure of interferon-free therapy}

GT1a-infected patients who have failed dual DAA therapy (SOF/LDV, ASV + DCV or GZR/EBR) should be tested for RAV before planning retherapy. The procedure is not necessary in GT1b-infected patients who can be considered for OBV/PTV/r $+\mathrm{DSV} \pm \mathrm{RBV}$ retherapy without RAV testing. Failure of OBV/PTV/r $+\mathrm{DSV} \pm \mathrm{RBV}$ treatment should be followed by RAV testing for the purpose of planning future retherapy based on drugs demonstrating a higher genetic bar- rier than currently available agents (especially NS5A inhibitors) [7, 10, 15-17].

\section{HCV genotype 2 infection}

Combined therapy with SOF + RBV is used in previously untreated patients for 12 weeks. The therapy should be extended to 24 weeks in patients with cirrhosis, after liver transplantation, with high baseline viraemia and previous unsuccessful treatment with PegIFN $\alpha+$ RBV [24].

Following the failure of SOF + RBV treatment, patients with F3/4 fibrosis and after liver transplantation may be considered for a 24-week SOF + DCV + RBV regimen, bearing in mind that the therapeutic management is not currently reimbursed in Poland [7].

The regimen recommended for the treatment of children older than 3 years is PegIFN $\alpha+$ RBV for 24 weeks. The same drug therapy may also be considered in previously untreated adults with mild liver fibrosis [22, 23].

\section{HCV genotype 3 infection}

Triple drug therapy with SOF + PegIFN $\alpha+$ RBV used for 12 weeks ensures the highest efficacy of treatment in patients infected with HCV genotype 3. Patients with contraindications (Table 3 ), interferon intolerance (Table 4 ) or after liver transplantation should follow the SOF + RBV regimen for 24 weeks. In cases

Table 3. Contraindications to interferon $\alpha$-based therapy

Interferons $\alpha$ should not be used in the following circumstances:

History of hypersensitivity to interferons or any of the excipients

Decompensated cirrhosis

Hepatitis or another disease of autoimmune aetiology

Status post transplantation of liver or any other organ

Patients approved for liver transplantation

Pregnancy

Severe (especially unstable) heart disease

Generalized atherosclerosis

Chronic respiratory failure

Metabolic syndrome and difficult-to-treat diabetes, following consultation with an endocrinologist

Depression, suicidal ideation or attempts documented by a psychiatric evaluation

Thyroid diseases involving abnormal thyroid-stimulating hormone (TSH) levels

Anaemia

Thrombocytopenia $<90,000 / \mu l$

Absolute neutrophil count $<1,500 / \mu l$ 
Table 4. Criteria of interferon intolerance

\begin{tabular}{l}
\hline Hypersensitivity to interferon or any of the excipients \\
\hline Autoimmune disease \\
\hline Exacerbation of a previously existing comorbidity \\
\hline Decrease in initial body weight by more than $20 \%$ \\
\hline Depression, suicidal ideation or attempts \\
\hline Thyroid dysfunction \\
\hline Haemoglobin concentration $<8.5 \mathrm{mg} \%$ \\
\hline Thrombocytopenia $<50,000 / \mu \mathrm{l}$ \\
\hline Absolute neutrophil count $<500 / \mu \mathrm{l}$ \\
\hline
\end{tabular}

involving the development of PegIFNa intolerance during SOF + PegIFN $\alpha+$ RBV therapy, an attempt must be made to reduce the dose of PegIFN $\alpha$, and if the strategy proves unsuccessful, interferon should be discontinued, and treatment with SOF + RBV should be maintained until week 24 [24].

Following the failure of all therapeutic options presented above, patients with advanced liver fibrosis can be considered for SOF/LDV + RBV for 24 weeks, SOF $+\mathrm{DCV}+\mathrm{RBV}$ for 24 weeks or GZR/EBR + SOF for 12 weeks $[7,25]$.

The regimen recommended for the treatment of children older than 3 years is PegIFN $\alpha+$ RBV for 24 weeks. The same drug therapy may also be considered in previously untreated adults with mild liver fibrosis $[22,23]$.

\section{HCV genotype 4 infection}

The optimal therapeutic regimens in HCV genotype 4 -infected patients, both previously untreated and following unsuccessful PegIFN $\alpha+$ RBV treatment, is OBV/ $\mathrm{PTV} / \mathrm{r}+\mathrm{RBV}$ or SOF/LDV $\pm \mathrm{RBV}$. However, if their initiation is not possible, SOF + PegIFN + RBV or SOF + RBV should be considered. If the above options fail, patients should wait for new therapeutic approaches.

\section{Ombitasvir/paritaprevir/ritonavir}

OBV/PTV/r should be continued in combination with RBV for 12 weeks, and in patients with cirrhosis extended to 24 weeks. OBV/PTVr + RBV therapy should not be used in patients with advanced liver failure and Child-Pugh score C. Patients with a history of hepatic encephalopathy incidents or ascites, as well as patients with Child-Pugh scores B and C, can be considered for treatment under close monitoring in medical centres with experience in the treatment of patients with decompensated cirrhosis. In patients after liver transplantation, $\mathrm{OBV} /$ $\mathrm{PTV} / \mathrm{r}+\mathrm{RBV}$ should be continued for 24 weeks [14-16].

\section{Sofosbuvir/ledipasvir}

Previously untreated patients without cirrhosis should continue SOF/LDV treatment for 12 weeks. Reducing the duration of therapy to 8 weeks does not seem to be fully justified by clinical practice. Patients who have failed previous therapy (including triple interferon-based therapy), with cirrhosis (also decompensated), and after liver transplantation should receive SOF/LDV + RBV for 12 weeks, and if RBV cannot be used, SOF/LDV treatment should be extended to 24 weeks [17].

\section{Grazoprevir + elbasvir}

GZR/EBR therapy in GT4-infected patients should last 12 weeks. However, patients with a history of ineffective interferon treatment should use GZR/EBR + RBV for 16 weeks [21].

\section{Pegylated interferon $\alpha+$ ribavirin}

Pegylated interferon $\alpha+$ ribavirin for a total of 48 weeks is a regimen recommended in children after 3 years of age $[22,23]$.

\section{Other therapeutic options}

$\mathrm{SOF}+\mathrm{PegIFN} \alpha+\mathrm{RBV}$ is used for 12 weeks regardless of the stage of liver fibrosis. Patients with contraindications (Table 3), interferon intolerance (Table 4) or after liver transplantation should take SOF + RBV for 24 weeks. In cases involving the development of PegIFN $\alpha$ intolerance during SOF + PegIFN $\alpha+$ RBV therapy, an attempt must be made to reduce the dose of PegIFN $\alpha$, and if the strategy proves unsuccessful, interferon should be discontinued, and treatment with SOF + RBV should be maintained until week 24 [24].

\section{Infection with HCV genotypes 5 and 6}

\section{Sofosbuvir/ledipasvir}

Previously untreated patients without cirrhosis should continue SOF/LDV treatment for 12 weeks. Reducing the duration of therapy to 8 weeks does not seem to be fully justified by clinical practice. Patients who have failed previous therapy (including triple interferon-based therapy), with cirrhosis (also decompensated), and after liver transplantation should receive SOF/LDV + RBV for 12 weeks, and if RBV cannot be used, SOF/ LDV treatment should be extended to 24 weeks [17].

An alternative regimen is SOF + PegIFN $\alpha+\mathrm{RBV}$ for a total of 12 weeks regardless of the stage of liv- 
er fibrosis. Patients with contraindications (Table 3), interferon intolerance (Table 4) or after liver transplantation should follow the SOF + RBV regimen for 24 weeks. In cases involving the development of PegIFN $\alpha$ intolerance during SOF + PegIFN $\alpha+$ RBV therapy, an attempt must be made to reduce the dose of PegIFN $\alpha$, and if the strategy proves unsuccessful, interferon should be discontinued, and treatment with SOF + RBV should be maintained until week 24 [22].

\section{Disclosure}

Waldemar Halota: Consultancy/Advisory Board/ Speaker/Grant: AbbVie, Alfa Wasserman, BMS, Gilead, Janssen, Merck, Roche.

Robert Flisiak: Consultancy/Advisory Board/ Speaker/Grant: AbbVie, Alfa Wasserman, BMS, Gilead, Janssen, Merck, Roche.

Anna Boroń-Kaczmarska: Consultancy/Advisory Board/Speaker/Grant: AbbVie, BMS, Gilead, Janssen, Merck, Roche.

Jacek Juszczyk: Consultancy/Speaker: AbbVie, Alfa Wasserman, BMS, Gilead, Merck.

Małgorzata Pawłowska: Consultancy/Advisory Board/Speaker/Grant: AbbVie, Alfa Wasserman, BMS, Gilead, Janssen, Merck, Roche.

Krzysztof Simon: Consultancy/Advisory Board/ Speaker/Grant: AbbVie, Actelion, Alfa Wasserman, Bayer, Baxter, BMS, Gilead, Intercept, Janssen, Merck, Roche, Tobira.

Krzysztof Tomasiewicz: Consultancy/Advisory Board/Speaker/Grant: AbbVie, Alfa Wasserman, BMS, Gilead, Janssen, MSD, Roche.

Piotr Małkowski: No conflict of interest.

\section{References}

1. Wedemeyer H, Dore GJ, Ward JW. Estimates on HCV disease burden worldwide - filling the gaps. J Viral Hepatitis 2015; 22 (Suppl 1): 1-5.

2. Flisiak R, Halota W, Horban A, et al. Analysis of risk factors related to HCV infection in Poland. Eur J Gastroenterol Hepatol 2011; 23: 1213-1217.

3. Flisiak R, Halota W, Tomasiewicz K, et al. Forecasting the disease burden of chronic hepatitis $\mathrm{C}$ virus in Poland. Eur J Gastroenterol Hepatol 2015; 27: 70-76.

4. Panasiuk A, Flisiak R, Mozer-Lisewska I, et al. Distribution of HCV genotypes in Poland. Przegl Epidemiol 2013; 67: 11-16.

5. Westbrook RH, Dusheiko G. Natural history of hepatitis C. J Hepatol 2014; 61 (1 Suppl): S58-68.

6. Halota W, Flisiak R, Boroń-Kaczmarska A, et al.; Polish Group of HCV Experts. Recommendations for the treatment of hepatitis C Polish Group of HCV Experts - 2015. Clin Exp Hepatol 2015; 3: 97-104.

7. European Association for Study of Liver. EASL recommendations on treatment of hepatitis C 2015. J Hepatol 2015; 63: 199-236.

8. Jaroszewicz J, Flisiak-Jackiewicz M, Lebensztejn D, et al. Current drugs in early development for treating hepatitis $\mathrm{C}$ vi- rus-related hepatic fibrosis. Expert Opin Investig Drugs 2015; 24: 1229-1239.

9. www.hepatitisc.uw.edu/page/clinical-calculators/apri

10. Lontok E, Harrington P, Howe A, et al. Hepatitis C virus drug resistance-associated substitutions: State of the art summary. Hepatology 2015; 62: 1623-1632.

11. Wasiak D, Małkowski P. Wytyczne leczenia raka wątrobowokomórkowego (HCC). Med Sci Mon Rev Hepatology 2013; 13: 112-115.

12. Badri P, Dutta S, Coakley E. Pharmacokinetics and dose recommendations for cyclosporine and tacrolimus when coadministered with ABT-450, ombitasvir, and dasabuvir. Am J Transplant 2015; 15: 1313-1322.

13. www.hep-druginteractions.org

14. Flisiak R, Janczewska E, Wawrzynowicz-Syczewska M, et al. Efficacy and safety of paritaprevir/r/ombitasvir/dasabuvir +/ribavirin in genotype $1 \mathrm{HCV}$ infected patients treated in real life settings (AMBER study). J Viral Hepatitis 2015; 22 (Suppl 3): 4.

15. Viekirax, Charakterystyka Produktu Leczniczego.

16. Exviera, Charakterystyka Produktu Leczniczego.

17. Harvoni, Charakterystyka Produktu Leczniczego.

18. Daklinza, Charakterystyka Produktu Leczniczego.

19. Kumada H, Suzuki Y, Ikeda K, et al. Daclatasvir plus Asunaprevir for chronic HCV genotype 1b infection. Hepatology 2014; 59: 2083-2091.

20. Manns M, Pol S, Jacobson IM, et al. All-oral daclatasvir plus asunaprevir for hepatitis $\mathrm{C}$ virus genotype $1 \mathrm{~b}$ : a multinational, phase 3, multicohort study. Lancet 2014; 384: 1597-1605.

21. Zapatier, FDA prescribing information.

22. Pegasys, Charakterystyka Produktu Leczniczego.

23. Pegintron, Charakterystyka Produktu Leczniczego.

24. Sovaldi, Charakterystyka Produktu Leczniczego.

25. Zapatier, Product monograph including patient medication information, Canada. 